Revista Colombiana de Obstetricia y Ginecología Vol. 57 No. 1 • 2006 • (19-26)

INVESTIGACIÓN ORIGINAL

\title{
CREENCIAS, ACTITUDES Y VIVENCIAS MÁGICAS ALREDEDOR DE LA MENSTRUACIÓN ENTRE LAS MUJERES DE BUCARAMANGA, COLOMBIA
}

\section{Beliefs, attitudes and magical experiences regarding menstruation among women from Bucaramanga, Collombia}

Miguel Ángel Alarcón- Nivia, M.D.*, Miguel Ángel Alarcón-Amaya, M.D.**, Lizzeth Blanco-Fuentes, M.D.**

Recibido: febrero 1/2006 - Revisado: marzo 23/2006 - Aceptado: marzo 30/2006

“...sed nihil facile reperiatur mulierum profluvio magis monstrificum...”

Plinio EI Viejo: Libro VII, párrafo 64, Naturalis Historiae

\section{RESUMEN}

En este estudio mostramos el resultado de la entrevista a 1.207 mujeres sobre su percepción de los malestares más frecuentes asociados a la menstruación, las prohibiciones relacionadas con este fenómeno fisiológico, y exponemos la vigencia de las creencias mágicas positivas (poderes curativos) y negativas (efectos nocivos) en cada persona que recibe sangre menstrual untada o tomada.

Palabras clave: menstruación, síndrome premenstrual, creencias tradicionales, Colombia

\section{SUMMARY}

The article presents the results of interviews conducted with 1,207 women concerning their perception of

\footnotetext{
* Profesor asociado Departamento de Ginecología y Obstetricia, Universidad Industrial de Santander (UIS), Correo Electrónico: alarcon@intercable.net.co

** Médicos cirujanos UIS
}

the most common symptoms associated with menstruation and prohibitions related to this physiological phenomenon. It depicts current magical beliefs, either beneficial (healing powers) or negative ones (morbid powers), in each person receiving menstrual blood, whether spread over their body or ingested.

Key words: menstruation, premenstrual syndrome, traditional beliefs, Colombia.

\section{INTRODUCCIÓN}

Muchos años atrás tuve la fortuna de leer un resumen del Naturalis Historiae, ${ }^{l}$ una serie de 37 libros escritos en Roma hacia el año 65 del siglo I, por el enciclopedista e historiador Gaius Plinius Cecilius Secundus. El libro VII menciona el lóbrego influjo de las mujeres menstruantes y las terribles consecuencias sobre las cosechas, los alimentos, los fenómenos naturales, etc. que se exponen a la influencia mágica del fluido menstrual. De esa 
lectura quedó latente la inquietud de investigar sobre el tema en Bucaramanga, ciudad de 800.000 habitantes, ubicada al nor-oriente de Colombia. Quien lea este documento y lo compare con la "Historia Natural" de Plinio el Viejo, encontrará un asombroso parecido entre las creencias mágicas de las mujeres interrogadas hace más de 1.900 años y las entrevistadas por nosotros.

"Pero no se hallará con facilidad nada más prodigioso que el flujo menstrual de las mujeres. La proximidad de una mujer en este estado hace agriar el mosto; a su contacto, los cereales se convierten en estériles, los injertos mueren, las plantas de los jardines se secan, los frutos de los árboles donde ella está sentada caen; el resplandor de los espejos se enturbian nada más que por su mirada; el filo del acero se debilita, el brillo del marfil desaparece, los enjambres de abejas mueren; incluso el bronce y el hierro se oxidan inmediatamente y el bronce toma un olor espantoso; en fin, la rabia le entra a los perros que prueban de dicho líquido y su mordedura inocula un veneno sin remedio. Este flujo tan curioso y tan pernicioso aparece todos los treinta días en la mujer, $y$, con más intensidad todos los tres meses". Plinio (Hist. Nat., VII, numerales 64-66). Quizás la curiosidad de Plinio por este tema fue motivada por Aristóteles, quien en el siglo III A.C. escribía en el primer libro de La Historia de los Animales ${ }^{2}$ sobre las características de la menstruación en la mujer y en las hembras de los animales.

El periodo que precede la llegada de la regla y los días que dura la misma, inducen cambios en la alimentación, en los hábitos de aseo, en la forma de vestirse, en las actividades laborales, en la vida sexual, etc.

El objetivo de este estudio era indagar a profundidad sobre los cambios en las actitudes y en las vivencias mágicas alrededor de la menstruación entre las mujeres de todos los estratos económicos y niveles de instrucción de esta ciudad.

\section{MATERIALES Y MÉTODOS}

Esta investigación fue realizada mediante entrevista personal a 1.320 mujeres de 15 a 49 años de edad, del área metropolitana de Bucaramanga durante
10 años, desde 1994 hasta el 2004, exceptuando el año 1996. El promedio de entrevistadas por año fue de 132. Se desecharon 113 formularios por presentar más del 25\% de espacios en blanco, es decir, sin respuestas. Por esta razón la muestra final fue de 1.207 mujeres. Los cuestionarios definitivos se elaboraron con preguntas abiertas, modificadas y adaptadas de los cuestionarios Menstrual Symptom Questionnaire $M S Q,{ }^{3}$ (diseñado por Margarette Chesney y Donald Tasto, del Departamento de Psicología de la Universidad del Estado en Fort Collins, Colorado, en 1975), del Menstrual Attitude Questionnaire, MAQ (diseñado por Jeanne Brooks, Diane Ruble y Anne Clark de la Universidad de Princenton, Nueva Jersey, en 1980) ${ }^{4}$ y del Menstrual Distress Questionnaire, MDQ-9 (diseñado en 1968 por Rudolph H. Moos, profesor de psiquiatría del Stanford University Medical Center). ${ }^{5}$ Inicialmente se emplearon solamente los cuestionarios mencionados, pero durante el estudio piloto inicial se vió la necesidad de introducir cambios para favorecer nuevas respuestas de las entrevistadas. Por esta razón se recalca que los cuestionaros MSQ, MAQ y MDQ-9, fueron tomados como apoyo, pero adaptados a las necesidades del estudio.

Los datos se recogieron en diferentes partes de la ciudad para obtener información de todos los niveles de instrucción y de todos los estratos económicos. Además de los autores, los entrevistadores fueron estudiantes de medicina del grupo de investigación de ginecología y algunos estudiantes que cursaban cada vez el semestre de ginecología y obstetricia de la Universidad Industrial de Santander. En la Tabla 1 se muestran algunas características demográficas de las mujeres entrevistadas. Cada pregunta permitía responder más de un ítem, o agregar otro originado por la entrevistada, por lo cual el número de respuestas sobrepasa al número de encuestas. Los porcentajes mostrados se calcularon del número total de respuestas obtenidas en cada pregunta. El análisis estadístico y la Figura 1 se hicieron con el programa Stata y con el programa Excel. 


\begin{tabular}{|l|c|c|c|c|c|c|}
\multicolumn{5}{|c|}{ Tabla 1. Características sociodemográficas de las pacientes encuestadas acerca de las } \\
creencias de la menstruación en Bucaramanga, Colombia 1994-2004 \\
\hline Estrato/Edad (años). & $\mathbf{n}$ & $\%$ & & Escolaridad & n por grupos \\
\hline Estratos I y II & 371 & 23,00 & & Ningún tipo de estudio & 39 \\
\hline Estrato III & 677 & 41,97 & Primaria o & Primaria incompleta & 300 & 55,3 \\
\hline Estrato IV & 399 & 21,01 & informal & Primaria completa & 278 & 13 \\
\hline Estratos V y VI & 226 & 14,01 & & Otras formas de instrucción & 113 \\
\hline Edad 15-20 & 321 & 24,3 & Secundaria & Secundaria incompleta & 376 \\
\hline Edad 21-30 & 345 & 26,1 & Secundaria completa & 112 & 36,9 \\
\hline Edad 31-40 & 334 & 25,3 & Estudios & Universitaria completa & 21 \\
\hline Edad 41-49 & 320 & 24,2 & superiores & Universitaria incompleta & 81 \\
\hline
\end{tabular}

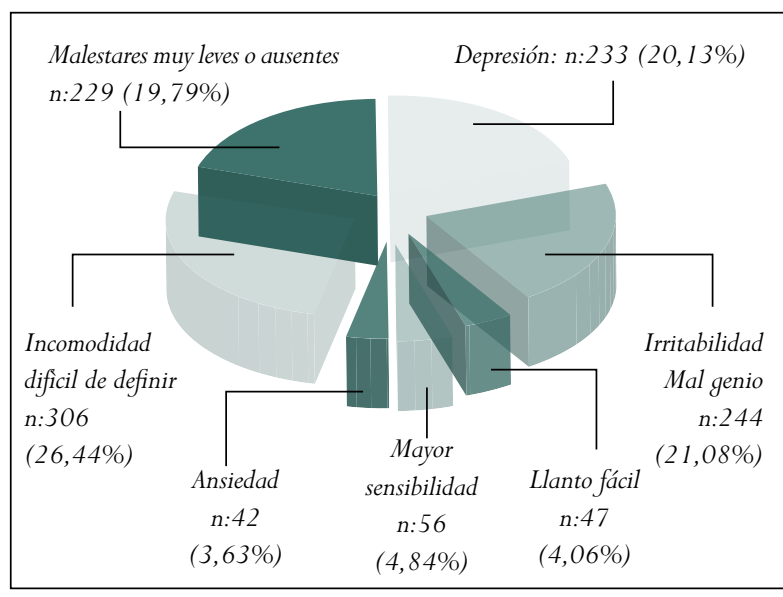

Figura 1. Frecuencia de los malestares premenstruales o menstruales. Los porcentajes se calcularon del total de 1.157 respuestas.

\section{RESULTADOS}

\section{Los nombres coloquiales actuales dados a la menstruación}

Se obtuvieron los nombres coloquiales más frecuentes, publicados primero por Flórez en $1.969^{6}$ y luego actualizados por la Revista Colombiana de Obstetricia y Ginecología en el año 2.005. ${ }^{7}$

\section{Los malestares relacionados con el periodo menstrual}

En la Figura 1 se muestran los malestares más frecuentemente percibidos por las mujeres incluidas en esta investigación.

\section{Los cuidados corporales y de belleza}

Las respuestas relacionadas con los cuidados especiales que debe tener una mujer durante los días de la menstruación son expuestos en la Tabla 2.

\section{Los alimentos o bebidas que no deben consumir durante la regla}

El 68,1\% de las respuestas mencionan que consumir alimentos ácidos cortan la regla, consumir ácidos aumentan la cantidad de sangre, los ácidos pueden enloquecer a la mujer, tomar leche esos días le da mal olor a la sangre, comer aguacate, banano o licor indispone a la mujer en su salud. El 28,4\% de las mujeres entrevistadas respondió que pueden tomar cualquier alimento o bebida sin efecto sobre su salud. El 3,5\% de las entrevistadas no respondieron esta pregunta.

\section{Efectos benéficos de tener la menstruación}

En general las mujeres entrevistadas perciben la llegada de la menstruación cada mes como un signo de bienestar corporal, como una forma de reafirmar la feminidad y como una demostración de juventud. El 17\% de las entrevistadas mencionaron que la mujer que está menstruando está inmune a la mordedura de serpientes. 


\begin{tabular}{|c|c|c|c|}
\hline & Tipo de cuidado & $\mathrm{n}$ & $\%$ por grupos \\
\hline \multirow{4}{*}{ Tener cuidados especiales } & Tener mucho aseo en el cuerpo & 389 & \multirow{4}{*}{41,6} \\
\hline & Bañarse con aguas aromáticas & 69 & \\
\hline & Hacerse aseo genital solamente & 176 & \\
\hline & Hacerse buen maquillaje por palidez & 387 & \\
\hline \multirow{5}{*}{ Precauciones } & No espicharse la cara porque se mancha & 248 & \multirow{5}{*}{54,1} \\
\hline & No cortarse las uñas porque se engruesan & 277 & \\
\hline & $\begin{array}{l}\text { No dejarse cortar el cabello ni cortárselo a nadie } \\
\text { porque se achila (se hace débil) }\end{array}$ & 201 & \\
\hline & $\begin{array}{l}\text { No bañarse esos días porque le corta la } \\
\text { menstruación o le dan cólicos }\end{array}$ & 299 & \\
\hline & $\begin{array}{l}\text { No dejarse cortar el cabello de una mujer que } \\
\text { tenga la menstruación }\end{array}$ & 301 & \\
\hline \multirow{2}{*}{ Indiferencia } & Ningún cuidado especial & 64 & \multirow{2}{*}{4,2} \\
\hline & No sé & 40 & \\
\hline \multicolumn{2}{|c|}{ Formularios sin respuesta a esta pregunta } & 9 & \\
\hline
\end{tabular}

\begin{tabular}{|c|c|c|c|}
\hline & & $\mathrm{n}$ & $\%$ por grupos \\
\hline \multirow{6}{*}{ Efectos físicos } & Se engorda & 670 & \multirow{6}{*}{70,5} \\
\hline & Le da un cáncer & 25 & \\
\hline & Le pueden aparecer tumores & 17 & \\
\hline & Le puede dar un derrame interno & 58 & \\
\hline & Produce abombamiento del abdomen & 99 & \\
\hline & Se le acumula la sangre por dentro y la intoxica & 348 & \\
\hline \multirow{2}{*}{ Efectos psicológicos } & Aumenta las preocupaciones & 269 & \multirow{2}{*}{19,2} \\
\hline & Da intranquilidad & 63 & \\
\hline \multirow{2}{*}{ Indiferentes } & Ninguno, no tiene efectos sobre la salud & 154 & \multirow{2}{*}{10,2} \\
\hline & No sé & 23 & \\
\hline \multicolumn{2}{|c|}{ Formularios sin respuesta a esta pregunta } & 19 & 0,1 \\
\hline \multicolumn{4}{|c|}{$\begin{array}{l}\text { Se obtuvieron } 1.726 \text { respuestas. El 70,5\% de las mismas manifiesta efectos indeseables en el cuerpo, causados por la } \\
\text { amenorrea. El 19,2\% menciona efectos psicológicos derivados de este trastorno. }\end{array}$} \\
\hline
\end{tabular}




\section{Los efectos de la falta de la menstruación}

La amenorrea es vista como una amenaza contra la salud. Se supone que la sangre que no se expulsa, se disemina por el cuerpo, y dadas sus propiedades tóxicas, causa diferentes trastornos físicos o psicológicos, mostrados en la Tabla 3.

\section{Los poderes curativos de la sangre menstrual de aplicación tópica}

Las respuestas más frecuentes, relacionadas con la aplicación de un poco de sangre untada sobre una parte del cuerpo, se muestran en la Tabla 4.

\section{La influencia dañina de la mujer durante los días de la regla}

\section{A. Los efectos sobre un niño pequeño o un animalito que es alzado o sobre una planta que es acariciada por una mujer durante los días de la menstruación:}

Hubo 1.351 respuestas. El 39,5\% de las mismas muestran temor o recelo con estos seres pues se teme que retarde o detenga su crecimiento. El 60,5 de las respuestas niegan algún efecto o responden "no sé".

\section{B. Alimentos o bebidas que no debe pre- parar durante la menstruación:}

Hubo 1.422 respuestas, el 73,8\% de las mismas tuvieron este tipo de respuestas: las cremas y pudines no crecen, las coladas se cortan, los alimentos que contengan leche se cortan, los alimentos a base de huevo se dañan, la carne se daña, los alimentos que contienen ácidos toman mal sabor. El 26,2\% de las respuestas negaban alguna influencia de la menstruación hacia los alimentos preparados.

\section{La vida sexual durante los días de la menstruación:}

A las preguntas relacionadas con su actitud hacia las relaciones sexuales durante los días del periodo menstrual, las respuestas obtenidas tuvieron tendencias de rechazo y de temor entre las mujeres de mayor edad. Las jóvenes mencionan indiferencia, aceptación o gusto. En la Tabla 5 se aprecian los resultados.

\section{Los efectos de la sangre menstrual que se le da a beber a un hombre}

Alrededor del 20\% de las encuestadas no responden o no creen que la sangre menstrual tenga algún

\begin{tabular}{|c|c|c|c|}
\hline & & $\mathrm{n}$ & $\%$ por grupo \\
\hline \multirow{6}{*}{$\begin{array}{l}\text { 1- Aceptan diferentes tipos } \\
\text { de beneficios de la sangre } \\
\text { menstrual }\end{array}$} & Le quita el acné al hombre & 211 & \multirow{6}{*}{43,64} \\
\hline & $\begin{array}{l}\text { Sirve para evitar "la picadura" } \\
\text { de serpiente }\end{array}$ & 323 & \\
\hline & Para el reumatismo & 110 & \\
\hline & Evita la caída del cabello & 89 & \\
\hline & Evita la indigestiwón & 28 & \\
\hline & Sirve para quien está mal de la digestión & 32 & \\
\hline \multicolumn{2}{|c|}{ 2- Niegan o ponen en duda los efectos benéficos de esta sangre } & 1.004 & 55,25 \\
\hline \multicolumn{2}{|c|}{ 3- No respondieron esta pregunta } & 20 & 1,10 \\
\hline
\end{tabular}




\section{Tabla 5. Percepción de las relaciones sexuales durante la menstruación}

\begin{tabular}{|c|c|c|c|}
\hline & Tipo de percepción & $\mathrm{N}$ & $\%$ por grupos \\
\hline \multirow{10}{*}{ Rechazo } & Se debe respetar a la mujer esos días & 359 & \multirow{10}{*}{64,7} \\
\hline & Es una falta de respeto & 139 & \\
\hline & Es una imprudencia & 118 & \\
\hline & Esos días son de descanso & 113 & \\
\hline & Es una falta de aseo & 112 & \\
\hline & Es antihigiénico & 84 & \\
\hline & Son muy incómodas & 49 & \\
\hline & Me dan asco & 93 & \\
\hline & Son cochinas & 15 & \\
\hline & Es una verdadera cochinada & 1 & \\
\hline \multirow{7}{*}{ Temor } & Aumentan la hemorragia del mes & 163 & \multirow{7}{*}{18,4} \\
\hline & Lastiman la vagina & 52 & \\
\hline & El hombre se enferma & 31 & \\
\hline & Al hombre le dan infecciones en el pene & 36 & \\
\hline & $\begin{array}{l}\text { Al hombre le va mal en los negocios/en el trabajo } \\
\text { en esos días }\end{array}$ & 13 & \\
\hline & El hombre se puede enloquecer por un tiempo & 9 & \\
\hline & Un hombre se puede volver loco & 4 & \\
\hline \multirow{10}{*}{ Aceptación y/o Gusto } & No hay ningún problema & 22 & \multirow{10}{*}{9,5} \\
\hline & Evitan el embarazo & 13 & \\
\hline & La sangre es lubricante & 8 & \\
\hline & Son agradables para el hombre & 16 & \\
\hline & A los hombres jóvenes no les importa & 37 & \\
\hline & Son placenteras & 18 & \\
\hline & Son muy excitantes & 19 & \\
\hline & Son super - excitantes & 8 & \\
\hline & Son ricas excepto el sexo oral & 15 & \\
\hline & Al hombre le gusta el sexo oral-genital en esos días & 3 & \\
\hline \multirow{2}{*}{ Indiferencia } & No tienen ningún atractivo & 42 & \multirow{2}{*}{7,3} \\
\hline & Me son indiferentes & 81 & \\
\hline \multicolumn{2}{|c|}{ Formularios sin respuesta a esta pregunta } & 11 & 0,1 \\
\hline
\end{tabular}

En el 72\% de los formularios había respuestas de rechazo o temor. En el 27\% de los mismos había aceptación, gusto o indiferencia. Se obtuvieron 1.673 respuestas. Los porcentajes muestran las tendencias de las mismas, discriminadas por grupos afines. 
efecto sobre el hombre que la toma en un líquido. Quienes aceptaron esta práctica mencionaron las siguientes bebidas como las más frecuentes: en jugo de mora, en un tinto, en coca-cola, en una aromática, en cualquier gaseosa, en una bebida alcohólica.

Un primer grupo de 693 entrevistadas respondieron que la sangre administrada de esta manera es benéfica (atrae más al hombre hacia la mujer y/o le da más apego sentimental). El segundo grupo, compuesto por 423 mujeres interrogadas, respondieron que esta práctica es perjudicial porque produce infecciones, puede llevar al hombre a la locura, le da indigestión, los vuelve impotentes o los deja como bobos. En 266 formularios adicionales, figuraba el concepto de un daño no especificado al receptor.

\section{DISCUSIÓN}

Desde el comienzo de los tiempos la menstruación ha tenido un halo de misterio para la humanidad. La llegada del fluido menstrual produce malestares físicos y psicológicos. ${ }^{8,9} \mathrm{La}$ abundante literatura que hay sobre el tema menciona que los días que preceden el sangrado y durante los días que dura el mismo, se producen cambios en la dieta, ${ }^{10}$ en el aseo corporal o en el aseo genital, ${ }^{11-14}$ en la forma de vestir, en la manera de elaborar los alimentos, en la forma de ofrecer algunos servicios a los clientes(si trabaja con alimentos o belleza), en las actitudes con las plantas y los niños y en la vida sexual. ${ }^{15}$ Se podría decir que no hay cultura humana en la cual no se tengan creencias folklóricas en torno a este proceso fisiológico. Las mujeres entienden la menstruación como un testigo de su fisiología normal, por lo cual la amenorrea o la excesiva cantidad de sangrado, las hace temer una enfermedad. ${ }^{16-17}$ Los resultados obtenidos en este estudio son concordantes con los datos de la literatura.

Otros investigadores en Colombia como Correa, ${ }^{18}$ Pinzón $^{19}$ y Uribe, ${ }^{20}$ se han interesado en el pasado por este tema. Múltiples autores de otras latitudes han incursionado en el tema de la magia y de las costumbres folclóricas de la menstruación. ${ }^{21-22}$ Los conocimientos científicos ganados alrededor de la menstruación normal y anormal en los últimos $a_{n} \operatorname{ss}^{23} \mathrm{y}$ en los anteriores veinte siglos para explicar los eventos fisiológicos o patológicos, han tenido que competir de manera pertinaz con las creencias populares basadas en la magia, en la religión y en los poderes sobrenaturales adquiridos por las mujeres que cada mes se cargan de energía negativa y dañan los alimentos, detienen el crecimiento de las plantas o ejercen mala influencia sobre sus compañeros sexuales. Sin embargo este extraño fluido, tiene su cara opuesta: la de los poderes benefactores como la protección de la mujer menstruante contra la mordedura de las serpientes, como pócima mágica que atrae al ser amado, como sustancia tópica para el tratamiento del reumatismo, del acné, etc.

\section{CONCLUSIONES}

Esta investigación muestra el poder milenario de la tradición oral, presente tanto en los grupos con bajo nivel de escolaridad, como en las mujeres con alto nivel económico y de instrucción universitaria. Los hallazgos de este estudio muestran una de las aristas de la magia en la vida cotidiana del siglo XXI, en gran parte de las mujeres de Bucaramanga. Las respuestas muestran la influencia negativa sobre aquello que crece (los niños, las plantas, el cabello, las uñas, el pudín); muestran el temor por el poder tóxico de la sangre que no sale del cuerpo de la mujer, o el efecto seductor y/o venenoso contra un hombre que recibe sangre oculta en una bebida. Gran parte de las creencias mágicas recogidas por Aristóteles, por el rey Juba de Mauritania y por Teofrasto, consultados por Plinio El Viejo, se mantienen sin grandes cambios desde los comienzos de la era cristiana, y quizás desde antes, y se detectan veinte siglos después en estudios como este.

Es fácil deducir que en las centurias venideras, la tradición oral y la intermediación de los astrólogos, numerólogos, brujos, etc. que utilizan la radio, la televisión y los medios escritos, mantendrán vivas las creencias en la medicina mágica, en los efectos 
sobrenaturales de la sangre menstrual, en los talismanes... pero en ausencia del oráculo de Delfos y de los consultorios sentimentales, habrá soluciones mágicas disponibles en las páginas web y en la telefonía satelital con modernos descendientes de Sibila, la pitonisa que servía de intermediaria entre Apolo y los hombres.

\section{AGRADECIMIENTOS}

A todos los estudiantes de Medicina de la U.I.S., que colaboraron en esta investigación, por su tiempo y dedicación para realizar las entrevistas.

\section{REFERENCIAS}

1. Plinius. Naturalis Historiae. Edited by Jan C, Mayhoff C. Published by K.G. Saur Verlag. Distributed by the University of Michigan Press in Canada and the United States; 1969.

2. Aristotle. History of animals. Books I-III. Harvard University Press, June 1984. (El original se escribió en el siglo III A.C.).

3. Chesney MA, Tasto DL. The effectiveness of behaviormodification with spasmodic and congestive dysmenorrhea. Behav Res Ther 1975;13:245-3.

4. Brooks-Gunn J, Ruble DN. The menstrual attitude questionnaire. Psychosom Med 1980;42:503-12.

5. Moos RH. The development of a menstrual distress questionnaire. Psychosom Med 1968;30:853-67.

6. Flórez L. Léxico del cuerpo humano en Colombia. Bogotá: Instituto Caro y Cuervo; 1969.

7. Alarcón-Nivia MA. Algunas consideraciones antropológicas y religiosas alrededor de la menstruación. Rev Colomb Obstet Ginecol 2005;56:35-45.

8. Richards M, Rubinow DR, Daly RC, Schmidt PJ. Premenstrual symptoms and perimenopausal depression. Am J Psychiatry 2006;163:133-7.

9. Martin VT, Wernke S, Mandell K, Ramadan N, Kao L, Bean J, et al. Symptoms of premenstrual syndrome and their association with migraine headache. Headache 2006;46:125-37.
10. Harvey LJ, Armah CN, Dainty JR, Foxall RJ, John Lewis $\mathrm{D}$, Langord NJ, et al. Impact of menstrual blood loss and diet on iron deficiency among women in the UK. Br J Nutr 2005;94:557-64.

11. Funkhouser E, Pulley L, Lueschen G, Costello C, Hook E 3rd, Vermund SH. Douching beliefs and practices among black and white women. J Womens Health Gend Based Med 2002;11:29-37.

12. El-Gilany AH, Badawi K, El-Fedawy S. Menstrual hygiene among adolescent schoolgirls in Mansoura, Egypt. Reprod Health Matters 2005;13:147-52.

13. Lichtenstein B, Nansel TR. Women's douching practices and related attitudes: findings from four focus groups. Women Health 2000;31:117-31.

14. James A Menstrual hygiene. A study of knowledge and practices. Nurs J India 1997;88:221-2.

15. Rempel JK, Baumgartner B. The relationship between attitudes towards menstruation and sexual attitudes, desires, and behavior in women. Arch Sex Behav 2003;32:155-63.

16. Oehler MK, Rees MC. Menorrhagia: an update. Acta Obstet Gynecol Scand. 2003;82:405-22.

17. Andrist LC, Hoyt A, Weinstein D, McGibbon C. The need to bleed: women's attitudes and beliefs about menstrual suppression. J Am Acad Nurse Pract 2004;16:31-7.

18. Correa A. Folklore de la menstruación. Antioquia Médica 1966;16(9-10).

19. Pinzón CE. Violencia y brujería en Bogotá. Boletín Cultural y Bibliográfico, No. 16. Bogotá: Banco de la República; 1988. p. 35-49.

20. Uribe CA, Martín E. Magia, brujería y violencia en Colombia. Colciencias y Universidad Nacional. Ponencia del IX Congreso de Antropología en Colombia (Popayán, julio de 2000).

21. Crawfurd R. Notes on the superstitions of menstruation. Lancet 1915;189:1331-6.

22. Snow LF, Johnson SM. Modern day menstrual folklore. Some clinical implications. JAMA 1977;237:2736-9.

23. Friberg B, Orno AK, Lindgren A, Lethagen S. Bleeding disorders among young women: a populationbased prevalence study. Acta Obstet Gynecol Scand 2006;85:200-6. 